\title{
Medication Adherence/Persistence and Demographics of Japanese Dyslipidemia Patients on Statin-Ezetimibe as a Separate Pill Combination Lipid-Lowering Therapy - An Observational Pharmacy Claims Database Study -
}

\author{
Tatsuya Umeda, PhD; Ai Hayashi, PhD; Go Fujimoto; Yi Piao, PhD; \\ Nobutomo Matsui, BSc; Shigeru Tokita, $\mathrm{PhD}$
}

\begin{abstract}
Background: This study aimed to identify potential predictors of medication adherence and persistence with statin-ezetimibe combinational lipid-lowering therapy (LLT) as a separate pill combination in a real-world setting in Japan.

Methods and Results: Patients newly switched to statin-ezetimibe combinational LLT from statin monotherapy were identified within a Japanese national pharmacy claims database during January 2015 to April 2018. Adherence and persistence were measured by the proportion of days covered (PDC), time to treatment discontinuation and persistence rate at 1 year. A stepwise multivariate logistic regression model and Cox proportional hazards regression model were used to explore potential predictors associated with adherence and persistence, respectively. Among 6,921 patients, $71.9 \%$ were adherent (PDC $\geq 80 \%$ ), and $83.6 \%$ were persistent at 1 year after initiation. Patients aged $\leq 54$ years and $\geq 75$ years were prone to be more non-adherent. Secondary prevention was associated with better adherence and longer persistence. Concomitant use of medications for depression/anxiety was associated with shorter persistence, whereas use of antihypertensive drugs was associated with better adherence and persistence.
\end{abstract}

Conclusions: Age, concomitant use of certain classes of medications (or the existence of these diseases) and secondary prevention were associated with adherence and persistence of statin-ezetimibe combinational LLT. Given that dyslipidemia is a chronic disease requiring life-long control, active interventions are required for patients with poor adherence and persistence.

Key Words: Adherence; Dyslipidemia; Ezetimibe; Risk factors; Statins

$\mathbf{S}$ tatin monotherapy is the recommended first-line treatment for controlling low-density lipoprotein cholesterol (LDL-C), followed by combination therapy of a statin with other lipid-lowering medicines such as ezetimibe or colestimide. This is well specified in the Japan Atherosclerosis Society (JAS) guidelines, as well as the USA and European guidelines. ${ }^{1-3}$ Dyslipidemia is a known risk factor for cardiovascular disease (CVD). Reduction of plasma LDL-C is crucial for preventing CVD events and clinical trials suggest a $1.0 \mathrm{mmol} / \mathrm{L}$ reduction in LDL-C is associated with reductions of $10 \%$ in all-cause death, $24 \%$ in major coronary events and $15 \%$ in stroke events. ${ }^{4,5}$

Adherence and persistence to statin therapy are reported to be lower in real-world clinical settings ${ }^{6-9}$ compared with well-controlled patients in the clinical trial setting where participants are actively managed for treatment compliance. Low adherence to medication can lead to poor control of LDL-C, ${ }^{9-12}$ resulting in a poor prognosis for CVD. ${ }^{13}$ Consequently, the importance of adhering to lipid-lower- ing therapy (LLT) for the management of CVD is emphasized in the treatment guidelines. In order to provide the most optimal patient support and maximize the efficacy of treatment, it is important to identify the characteristics of patient groups with poor adherence or persistence, or the potential predictors of poor adherence and persistence of patients who need strict management of LDL-C.

Factors that could affect medication adherence and discontinuation of statin monotherapy have been discussed in previous studies. ${ }^{14-17}$ Comorbidities have been shown to both positively and negatively affect adherence. For example, patients with dementia or respiratory disorders are at higher risk of being non-adherent with statin monotherapy, whereas patients with diabetes mellitus or hypertension showed a relatively low risk of medication discontinuation. ${ }^{14,17} \mathrm{~A}$ pooled meta-analysis of 45 studies suggested that patients on secondary prevention for CVD had better adherence than patients on primary prevention. ${ }^{14}$ Patients on secondary prevention may have a strong awareness of the impor-

Received January 25, 2019; revised manuscript received May 9, 2019; accepted May 31, 2019; J-STAGE Advance Publication released online July 5, 2019 Time for primary review: 46 days

Medical Affairs, MSD K.K., Tokyo (T.U., A.H., G.F., S.T.); Real World Insights, IQVIA Solutions Japan K.K., Tokyo (Y.P., N.M.), Japan

Mailing address: Tatsuya Umeda, PhD, Medical Affairs, MSD K.K., Kitanomaru Square, 1-13-12 Kudan-kita, Chiyoda-ku, Tokyo 102-8667, Japan. E-mail: tatsuya.umeda@merck.com

ISSN-1346-9843 All rights are reserved to the Japanese Circulation Society. For permissions, please e-mail: cj@j-circ.or.jp 


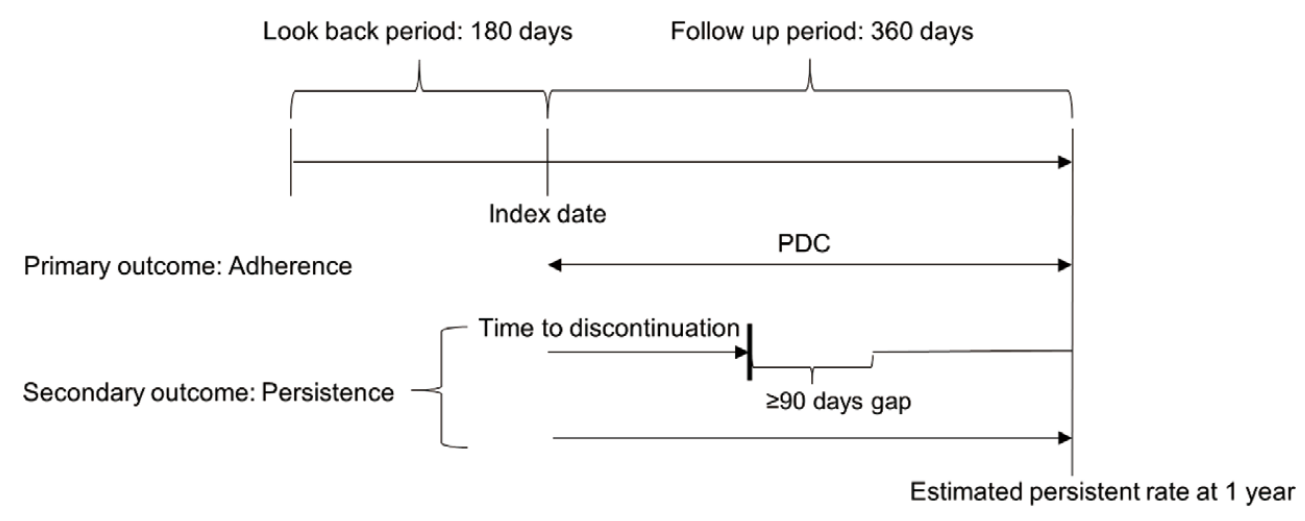

Figure 1. Study design. PDC, proportion of days covered.

tance of LDL-C control, given that they have experienced CVD events already, and it may affect patients' drug-taking behavior with statin therapy. Although the risk factors for discontinuation and non-adherence of statin monotherapy have been studied previously, it remains unclear which patients in Japan on statin-ezetimibe combinational LLT for better management of LDL-C are prone to poor adherence and persistence.

To our knowledge, this is the first study to explore the factors associated with the adherence and persistence of statin-ezetimibe LLT as a separate pill combination (SPC) regimen in a real-world setting. Utilizing one of the largest pharmacy claims database in Japan, this study aimed to identify potential predictors of medication adherence and persistence with the combination of statin-ezetimibe LLT in patients with dyslipidemia.

\section{Methods}

\section{Study Design and Subjects}

This was a retrospective study using Japanese national pharmacy dispensing claims data from January 2015 to April 2018 (Figure 1). Eligible patients were aged $\geq 20$ years and had been switched from statin monotherapy to the combination of statin with ezetimibe between July 2015 to April 2017. All statins on the Japanese market (pravastatin, simvastatin, fluvastatin, atorvastatin, pitavastatin and rosuvastatin) were considered. The index date was defined as the date when statin and ezetimibe were initially and concomitantly dispensed during July 2015 to April 2017 to the individual patient. Patients were included if they had another dispensing claim of statin-ezetimibe during the follow-up period after the index date, and had dispensing claims of statins as the only lipid-lowering agent during the lookback period. Patients were excluded if they could not be followed up for 1 year post-index and the 6-month pre-index period, and had any add-on lipid-lowering agents other than a statin or ezetimibe during the follow-up period.

\section{Data Source}

Prescription claims data were extracted from the IQVIA National Prescription Audit database. This database covers roughly $15.1 \%$ of all the national prescription records in 2016: 120 million prescriptions and 24.8 million patients.
The dispensing records were obtained from approximately 7,500 out-of-hospital pharmacies, which includes prescription information (e.g., generic names of therapeutic drugs, dosage forms, the date of dispensing, the number of days of supply) and patient characteristics (e.g., age, sex). The age distribution of the population in the database was similar to patients' demographics at the national level, based on surveys from the Ministry of Health, Labour and Welfare. ${ }^{18}$ All individual records were anonymized for secondary data analysis, and individuals could not be identified or linked to other data sources.

\section{Outcomes: Adherence and Persistence}

The primary outcome of this study was to evaluate the medication adherence of the dyslipidemic patients satisfying the study's criteria. The proportion of days covered (PDC) method was used to define adherence. PDC was calculated as the total number of dispensed days during a specified observation period divided by the number of observation days. ${ }^{19}$ Because the existence of multiple concomitant medications was under consideration of adherence measurements in this study, ${ }^{20,21}$ the number of days patients had both classes of medications (i.e., statin and ezetimibe) available during the 1-year post-index period was divided by 360 days for calculation of PDC. Patients with PDC $<80 \%$ were categorized as non-adherent, and those with PDC $\geq 80 \%$ were adherent. The threshold of $80 \%$ is commonly used for evaluating adherence to medications in previous studies. ${ }^{19}$

The secondary outcome was to evaluate persistence with combination therapy (statin-ezetimibe). Persistence was measured as (1) time to discontinuation, and (2) estimated persistence rate at 1 year. Time to discontinuation for an individual patient was defined as the number of days from the index date to the date when the patient discontinued the statin-ezetimibe combination regimen. Considering the gap between prescription date and supply, we regarded discontinuation as occurring when the first gap $\geq 90$ days without concomitant use of statin and ezetimibe appeared. The discontinuation date was determined as the date when the prescription of the previous combination regimen of statin-ezetimibe ended. Persistence was considered as censored and the time to discontinuation was from the index date to the end of follow-up in the time-to-event analysis if 
the $\geq 90$-day gap could not be identified before the end of follow-up. The $\geq 90$-day grace period has been used in many previous studies with regard to discontinuation of lipidlowering drugs. ${ }^{22,23}$ The estimated persistence rate at 1 year was the estimated proportion of patients with continued use of statin-ezetimibe at 1-year post-index.

\section{Potential Predictors}

The following variables were considered as potential predictors for adherence and persistence: age, sex, secondary prevention of CVD events, therapeutic area of interest, the number of concurrent therapeutic areas, the number of concurrent medications and the number of pills administered concurrently. Patients' concurrent therapeutic areas were estimated from the medication information and the drug's Anatomical Therapeutic Chemical (ATC) code prescribed during the look-back period. The therapeutic areas of interest to us were hypertension (ATC code: $\mathrm{C} 02, \mathrm{C} 03, \mathrm{C} 07$, C08, C09), diabetes mellitus (A10), chronic obstructive pulmonary disease/asthma (R03), gout (M04), depression/anxiety (N05B, N06A, N06C) and dementia (N06D, N07AB), which were either major comorbidities in patients with LLT or diseases associated with medication adherence. If patients had medications for coronary artery disease (ATC code: $\mathrm{B} 01, \mathrm{C} 01)$ before the index date, they were considered as secondary prevention of CVD events. The number of concurrent medications was determined by the medications provided and available at the index date, and the number of pills received concurrently was counted accordingly.

\section{Statistical Analysis}

Mean and standard deviation are reported as continuous variables, and frequency $(\mathrm{N})$ and percentage $(\%)$ are reported as categorical variables. The proportions of patients being adherent or non-adherent, and estimated persistence rate at 1-year post-index were summarized by characteristics. A stepwise multivariate logistic regression model was used to explore potential predictors associated with being adherent. Adjusted odds ratios (ORs) and $95 \%$ confidence intervals (CIs) are presented. For persistence, we used the KaplanMeier method to plot the time to discontinuation and performed a log-rank test to examine the difference between covariates, and calculate the estimated persistence rate at 1 year. A stepwise Cox proportional hazards regression model was used to explore potential predictors associated with time to discontinuation. Adjusted hazard ratios (HRs) and $95 \%$ CIs are presented. As the stepwise procedure, the covariates remaining in the model were determined statistically with a threshold for inclusion set at $\mathrm{P}<0.05$. The variable selection process was automatically performed by the statistical program. A significance level was set at $\alpha=0.05$ and all tests were two-sided. All the statistical analyses were performed using SAS version 9.4 (SAS Institute, Cary, NC, USA).

\section{Results}

\section{Patients' Characteristics}

A total of 24,661 patients received statin-ezetimibe as the SPC regimen during the period between January 2015 and April 2017. Of them, a total of 6,921 patients met the eligibility criteria and were identified as the study population who were newly initiated to a statin-ezetimibe regimen after being switched from statin monotherapy (Supplementary Figure 1). The mean age of the patients was 64.3 years, and
Table 1. Baseline Patients' Characteristics

$\quad$ Characteristic
Age (years)
Mean $\pm S D$
Age group, $\mathbf{n}(\%)$
$<45$
$45-54$
$55-64$
$65-74$
$\geq 75$

Sex, male, $\mathbf{n}(\%)$

Secondary prevention, yes, $\mathbf{n}(\%)$

Therapeutic area of interest, $\mathbf{n}(\%)$

Diabetes

Hypertension

Respiratory disease

Gout

Dementia

Depression/anxiety

No. of concurrent therapeutic areas

Mean \pm SD

Group, n (\%)

0

1

2

3

$\geq 4$

Overall

$(n=6,921)$

$64.3 \pm 12.1$

468 (6.8)

1,027 (14.8)

1,679 (24.3)

2,288 (33.1)

1,459 (21.1)

3,626 (52.4)

3,219 (46.5)

2,043 (29.5)

4,567 (66.0)

521 (7.5)

1,026 (14.8)

72 (1.0)

709 (10.2)

$1.8 \pm 1.2$

$1,196(17.3)$

$1,700(24.6)$

$2,140(30.9)$

$1,435(20.7)$

450 (6.5)

No. of concurrent medications

Mean \pm SD

$3.0 \pm 2.6$

Group, n (\%)

0

$1,352(19.5)$

1

$1,028(14.9)$

$969(14.0)$

$896(12.9)$

$839(12.1)$

$648(9.4)$

778 (11.2)

$6-7$

411 (5.9)

No. of pills received concurrently

Mean \pm SD

$4.1 \pm 4.0$

Group, n (\%)

0

$1,394(20.1)$

$902(13.0)$

$814(11.8)$

642 (9.3)

640 (9.2)

$491(7.1)$

$789(11.4)$

$723(10.4)$

$526(7.6)$

SD, standard deviation.

over half were aged $\geq 65$ years. Patients receiving secondary prevention accounted for $46.5 \%$ of the study population. Regarding comorbidities, nearly two-thirds of patients suffered from hypertension $(66.0 \%)$, followed by diabetes $(29.5 \%)$ and gout $(14.8 \%)$. The average number of concur- 


\begin{tabular}{|c|c|c|}
\hline Characteristic & $\begin{array}{c}\text { Adherent } \\
\text { (PDC } \geq \mathbf{8 0 \% )}, \mathrm{n}(\%)\end{array}$ & P value* \\
\hline Age group, n (\%) & & $<0.0001$ \\
\hline$<45$ & $300(64.1)$ & \\
\hline $45-54$ & $683(66.5)$ & \\
\hline $55-64$ & $1,229(73.2)$ & \\
\hline $65-74$ & $1,715(75.0)$ & \\
\hline$\geq 75$ & $1,048(71.8)$ & \\
\hline Sex, n (\%) & & $<0.0001$ \\
\hline Male & $2,685(74.0)$ & \\
\hline Female & $2,290(69.5)$ & \\
\hline Secondary prevention, $n(\%)$ & & $<0.0001$ \\
\hline Yes & $2,535(78.8)$ & \\
\hline No & $2,440(65.9)$ & \\
\hline \multicolumn{3}{|l|}{ Therapeutic area of interest, $\mathbf{n}(\%)$} \\
\hline Diabetes & $1,486(72.7)$ & 0.3067 \\
\hline Hypertension & $3,438(75.3)$ & $<0.0001$ \\
\hline Respiratory disease & $359(68.9)$ & 0.116 \\
\hline Gout & $784(76.4)$ & 0.0005 \\
\hline Dementia & $47(65.3)$ & 0.2102 \\
\hline Depression/anxiety & $491(69.3)$ & 0.1001 \\
\hline No. of concurrent therapeutic areas, $n(\%)$ & & $<0.0001$ \\
\hline 0 & $750(62.7)$ & \\
\hline 1 & $1,166(68.6)$ & \\
\hline 2 & $1,609(75.2)$ & \\
\hline 3 & $1,099(76.6)$ & \\
\hline$\geq 4$ & $351(78.0)$ & \\
\hline No. of concurrent medications, $n$ (\%) & & $<0.0001$ \\
\hline 0 & $848(62.7)$ & \\
\hline 1 & $686(66.7)$ & \\
\hline 2 & $698(72.0)$ & \\
\hline 3 & $674(75.2)$ & \\
\hline 4 & $647(77.1)$ & \\
\hline 5 & $510(78.7)$ & \\
\hline $6-7$ & $603(77.5)$ & \\
\hline$\geq 8$ & $309(75.2)$ & \\
\hline No. of pills received concurrently, $n(\%)$ & & $<0.0001$ \\
\hline 0 & $872(62.6)$ & \\
\hline 1 & $605(67.1)$ & \\
\hline 2 & $584(71.7)$ & \\
\hline 3 & $489(76.2)$ & \\
\hline 4 & $474(74.1)$ & \\
\hline 5 & $381(77.6)$ & \\
\hline $6-7$ & $609(77.2)$ & \\
\hline $8-10$ & $572(79.1)$ & \\
\hline$\geq 11$ & $389(74.0)$ & \\
\hline
\end{tabular}

${ }^{*} \mathrm{P}$ value calculated by Chi-square test. PDC, proportion of days covered.

rent medication and pills prescribed concomitantly at the index date were 3.0 and 4.1, respectively, covering a mean of 1.8 therapeutic areas (Table 1).

\section{Adherence by Characteristics}

Adherence stratified by patients' characteristics is shown in Table 2. It was found that $71.9 \%$ of the patients were adherent (i.e., PDC $\geq 80 \%$ ), and $28.1 \%$ were non-adherent (i.e., PDC $<80 \%$ ). The proportion of patients who were adherent was lower for females than for males. The proportion of patients being adherent was lower in the relatively younger group (age $<45$ years) and older group (age $\geq 75$ years), and was the highest in the mid-age group (age 65-74 years). In addition, a significantly higher proportion of patients receiving statin-ezetimibe therapy for secondary prevention were adherent compared with those receiving it for primary prevention ( $78.8 \%$ vs. $65.9 \% ; \mathrm{P}<0.05)$. In terms of comorbidities, a higher proportion of adherent patients 


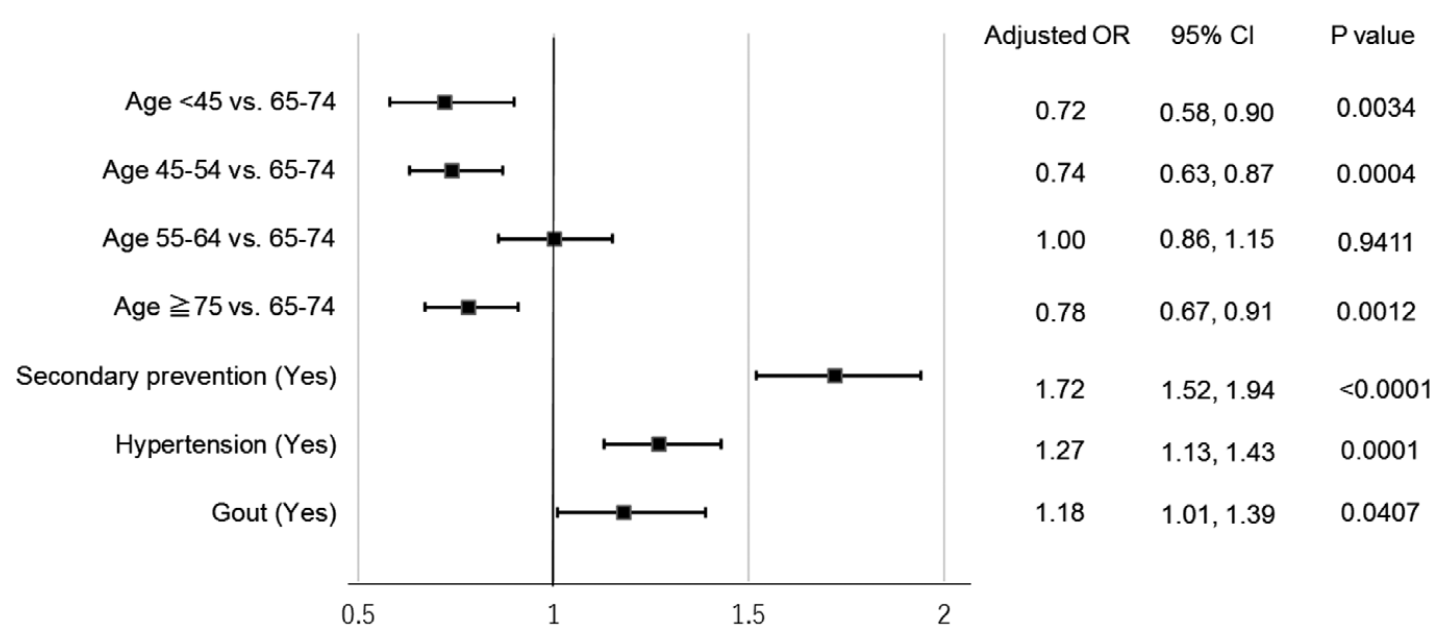

Figure 2. Odds ratios of factors associated with being adherent.

was observed among those with medications for gout (76.4\%), hypertension $(75.3 \%)$, or diabetes $(72.7 \%)$. On the other hand, the proportion of patients being adherent was lower among those prescribed treatment for dementia $(65.3 \%)$, depression/anxiety $(69.3 \%)$ or respiratory diseases $(68.9 \%)$. Patients without any other concomitant medications indicated for other therapeutic areas showed the lowest rate of being adherent. Patients tended to be more adherent with an increased number of concurrent therapeutic areas. In the group of patients prescribed concomitant medications in $\geq 4$ different estimated therapeutic areas, $78.0 \%$ were adherent, and $62.7 \%$ were adherent among patients without any concurrent therapeutic areas. Furthermore, the lowest proportion of being adherent $(62.7 \%)$ occurred for patients without any concurrent medications. The proportion of patients being adherent increased consecutively up to $78.7 \%$ among patients with 5 concomitant medications. However, when the number of concomitant medications was $>5$, the proportion of patients being adherent decreased. The trend for increasing adherence was similarly observed as the number of pills being prescribed concomitantly increased; however, the proportion of patients being adherent decreased in the group of patients prescribed $>10$ pills concurrently.

\section{Factors Associated With Adherence}

We investigated factors associated with adherence/non-adherence by multivariate logistic regression analysis (Figure 2). Compared with the age group 65-74 years, patients aged $\leq 54$ years or $\geq 75$ years were less adherent to the statinezetimibe therapy after adjusting other potential predictors. On the other hand, the adjusted OR $(95 \% \mathrm{CI})$ for being adherent was $1.72(1.52-1.94)$ for secondary prevention patients, using patients on primary prevention as the reference group. In addition, patients with medications for hypertension or gout were associated with better medication adherence, with OR $(95 \% \mathrm{CI})$ of 1.27 (1.13-1.43) and 1.18 (1.01-1.39), respectively. Factors including sex, the number of concurrent medications, the number of pills prescribed concomitantly, and the number of concurrent therapeutic areas were excluded by stepwise selection procedure in the logistic regression model.

\section{Persistence by Characteristics}

The proportion of patients being persistent on statin-ezetimibe therapy was evaluated at 1 year after the initiation of this combination prescription (Table 3). Similarly, the time to treatment discontinuation was evaluated over that time (Figure 3). Patients aged $<45$ years and those aged $\geq 75$ years discontinued treatment earlier than those aged 65-74 years. Early discontinuation ( $\approx 60$ days after the index date) was particularly observed in patients aged 20-44 years and patients with primary prevention. Moreover, patients prescribed with medications presumably indicated for dementia or depression/anxiety were prone to discontinue treatment earlier, while patients prescribed with medications for gout and hypertension were more likely to be persistent with treatment (Figure 3). The persistence rate was higher in patients with $\geq 4$ concurrent therapeutic areas than in patients without concurrent therapeutic areas. The estimated persistence rate in the overall population at 1 year after initiation of combination therapy was $83.6 \%$. Patients receiving statin-ezetimibe therapy for secondary prevention and also prescribed medications for gout, hypertension or diabetes showed higher persistence after 1 year of treatment.

\section{Factors Associated With Treatment Persistence}

Treatment discontinuation was further documented by the Cox proportional hazards model with adjusted HR (Figure 4). Patients aged $\geq 75$ years had a significantly higher risk of treatment discontinuation compared with those aged 65-74 years (HR: 1.28, 95\% CI: 1.09-1.51). Patients with secondary prevention had a significantly lower risk (HR: $0.70,95 \%$ CI: $0.61-0.80)$ of discontinuation than patients with primary prevention. Regarding the effect of comorbidities, patients treated with medications for hypertension were more likely to be persistent with treatment (HR: 0.76 , 95\% CI: 0.67-0.87). In contrast, patients treated with medications for depression/anxiety were associated with a higher risk of discontinuation (HR: $1.27,95 \% \mathrm{CI}: 1.06-1.51)$. The covariates of "sex", "the number of concurrent medications used" and "the number of pills received concurrently" were 


\begin{tabular}{|c|c|c|c|}
\hline Characteristic & $\begin{array}{l}\text { Persistence rate } \\
\text { at } 1 \text { year }(\%)\end{array}$ & $95 \% \mathrm{Cl}$ & P value* \\
\hline Overall & 83.6 & $82.7,84.5$ & \\
\hline Age group & & & 0.0005 \\
\hline$<45$ & 81.4 & $77.6,84.7$ & \\
\hline $45-54$ & 81.2 & $78.7,83.5$ & \\
\hline $55-64$ & 86.1 & $84.3,87.6$ & \\
\hline $65-74$ & 84.5 & $83.0,85.9$ & \\
\hline$\geq 75$ & 81.7 & $79.6,83.6$ & \\
\hline Sex & & & 0.0027 \\
\hline Male & 84.9 & $83.7,86.0$ & \\
\hline Female & 82.2 & $80.9,83.5$ & \\
\hline Secondary prevention & & & $<0.0001$ \\
\hline Yes & 87.1 & $85.9,88.2$ & \\
\hline No & 80.6 & $79.3,81.8$ & \\
\hline \multicolumn{4}{|l|}{ Therapeutic area of interest, $\mathbf{n}(\%)$} \\
\hline Diabetes & 84.7 & $83.0,86.2$ & 0.1184 \\
\hline Hypertension & 85.8 & $84.7,86.8$ & $<0.0001$ \\
\hline Respiratory disease & 81.2 & $77.6,84.3$ & 0.1082 \\
\hline Gout & 87.5 & $85.3,89.4$ & 0.0003 \\
\hline Dementia & 80.6 & $69.4,88.0$ & 0.5146 \\
\hline Depression/anxiety & 79.8 & $76.7,82.6$ & 0.0032 \\
\hline No. of concurrent therapeutic areas & & & $<0.0001$ \\
\hline 0 & 78.2 & $75.7,80.4$ & \\
\hline 1 & 82.4 & $80.5,84.1$ & \\
\hline 2 & 85.1 & $83.5,86.5$ & \\
\hline 3 & 85.6 & $83.7,87.4$ & \\
\hline$\geq 4$ & 89.1 & $85.9,91.7$ & \\
\hline No. of concurrent medications & & & $<0.0001$ \\
\hline 0 & 78.2 & $75.9,80.3$ & \\
\hline 1 & 79.7 & $77.1,82.0$ & \\
\hline 2 & 85.2 & $82.8,87.3$ & \\
\hline 3 & 85.7 & $83.2,87.8$ & \\
\hline 4 & 84.9 & $82.3,87.1$ & \\
\hline 5 & 88.3 & $85.5,90.5$ & \\
\hline $6-7$ & 87.9 & $85.4,90.0$ & \\
\hline$\geq 8$ & 84.7 & $80.8,87.8$ & \\
\hline No. of pills received concurrently & & & $<0.0001$ \\
\hline 0 & 78.2 & $75.9,80.3$ & \\
\hline 1 & 79.7 & $76.9,82.2$ & \\
\hline 2 & 84.4 & $81.7,86.7$ & \\
\hline 3 & 86.6 & $83.7,89.0$ & \\
\hline 4 & 84.8 & $81.8,87.4$ & \\
\hline 5 & 86.6 & $83.2,89.3$ & \\
\hline $6-7$ & 86.9 & $84.4,89.1$ & \\
\hline $8-10$ & 88.0 & $85.4,90.1$ & \\
\hline$\geq 11$ & 84.4 & $81.0,87.2$ & \\
\hline
\end{tabular}

${ }^{*} \mathrm{P}$ value calculated by log-rank test. $\mathrm{Cl}$, confidence interval.

automatically excluded by the stepwise selection procedure in the model.

\section{Discussion}

Study patients of interest were those prescribed statinezetimibe LLT in Japan, because of requiring more strict management of LDL-C control. We found that approxi- mately $71.9 \%$ of patients were adherent (PDC $\geq 80 \%$ ), and the overall persistence rate at 1 year was $83.6 \%$. These results suggested that management in dyslipidemia is still suboptimal and represents potential unmet clinical need for further improvement. Patients aged $\leq 54$ years or $\geq 75$ years were more prone to be non-adherent than patients aged 55-74 years, which was consistent with a systematic literature review showing that relatively younger and older 
A

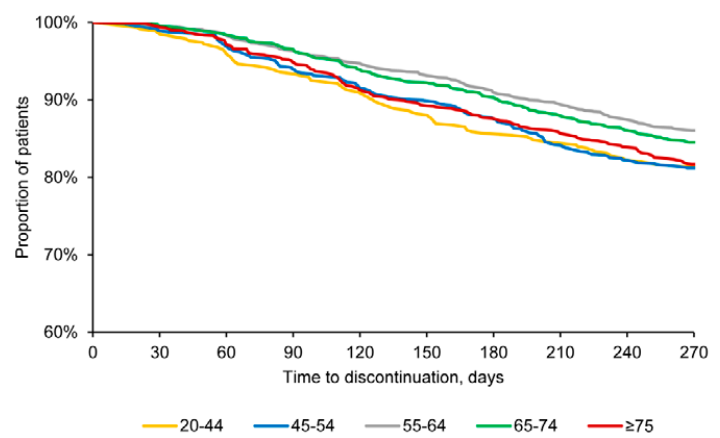

B

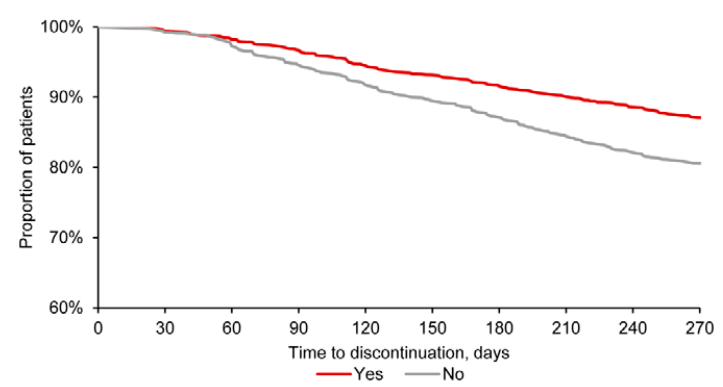

C

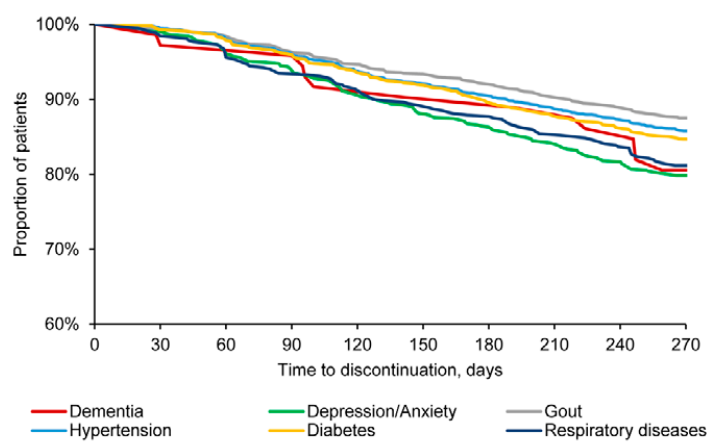

D

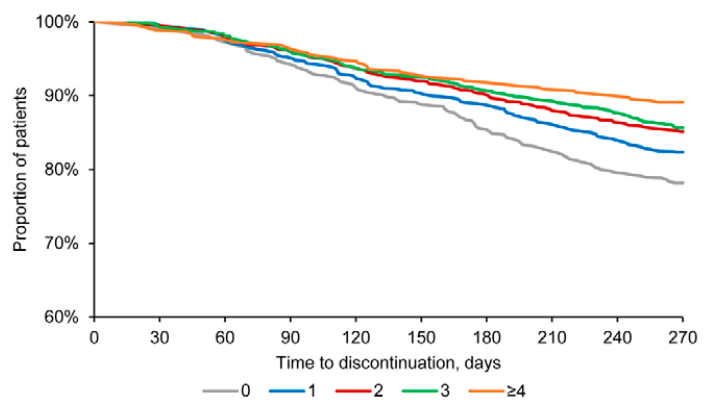

Figure 3. Kaplan-Meier analysis of time to discontinuation of statin-ezetimibe therapy. (A) Stratified by age groups; (B) stratified by secondary prevention (Yes/No); (C) stratified by therapeutic area of interest; (D) stratified by number of concurrent therapeutic areas.

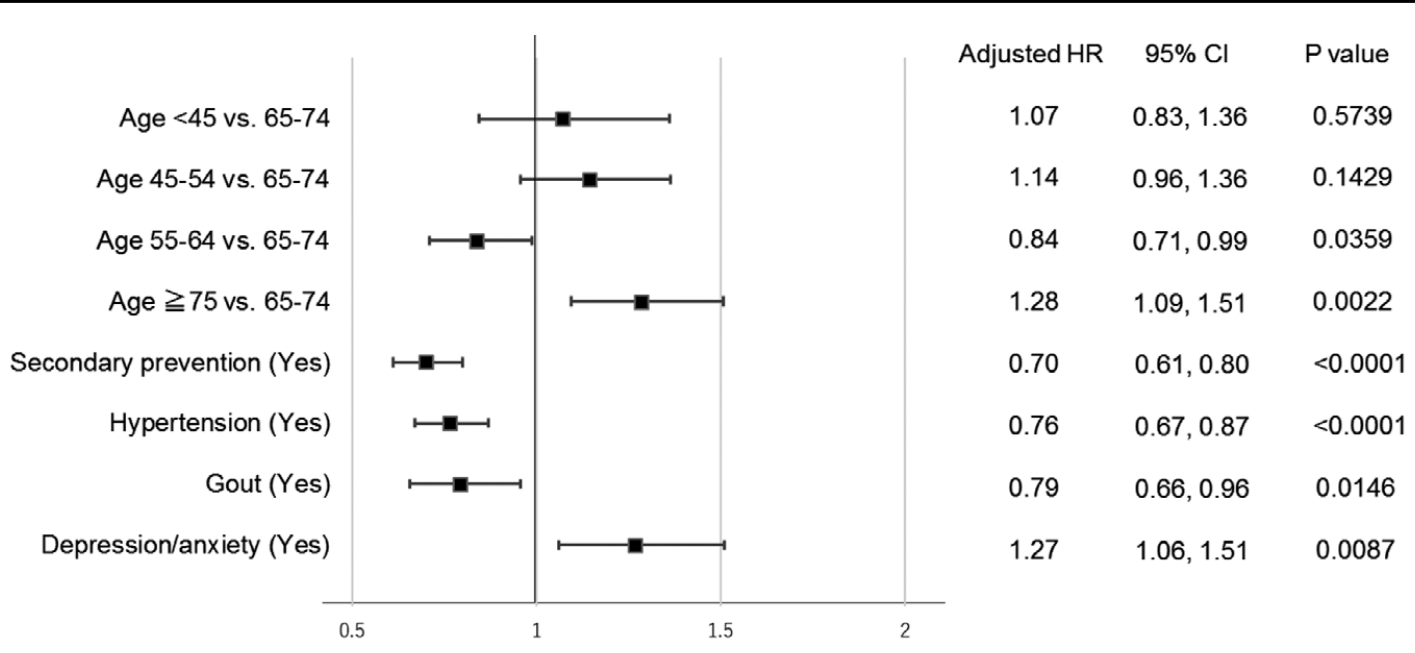

Figure 4. Hazard ratios of factors associated with discontinuation.

patients had poor adherence. ${ }^{17}$ It has been reported that younger patients have poor adherence because of lifestyle factors such as being busy at work and prone to forgetting to take medicine. ${ }^{24}$ Another previous study showed that younger patients on statin therapy had relatively poor persistence with medications. ${ }^{25}$ Our findings confirmed those trends shown in previously reported literature on the Japanese population.

We found that patients on secondary prevention were associated with better adherence and longer persistence than patients on primary prevention, who were associated with poor adherence and persistence. Our results are consistent 
with the pooled results from 45 articles in a meta-analysis study, which suggested $33 \%$ lower likelihood of non-adherence and $40 \%$ lower risk of discontinuation for patients on secondary prevention compared with patients on primary prevention. ${ }^{14}$ Another literature review suggested that the adherence is better in patients with a history of CVD events because of their appreciation of both the severity of potential complication and the perceived benefits. ${ }^{26} \mathrm{~A}$ study showed better adherence was associated with an approximately 3-fold higher probability of achieving target LDL-C level recommended in the treatment guidelines in 6 months $(\mathrm{OR}=2.74,95 \% \mathrm{CI}: 2.27-3.31){ }^{27}$ Thus, control of LDL-C is a major component in the secondary prevention strategy, which is aimed at patients with a history of CVD events. Consistent with this finding, our study suggested that patients on secondary prevention, who require stricter management of LDL-C, might be associated with adherence and persistence of statin-ezetimibe LLT because of their increased awareness of the importance of risk management. The IMPROVE-IT trial reported a benefit of statin-ezetimibe vs. statin alone in CVD risk reduction aligned with LDL-C reduction, in a median study period of 6 years. ${ }^{28}$ It suggests that persistence with medication in the long-term requires drug administration guidance and pharmacist intervention to maximize the benefit of the combination treatment in the control of CVD events. Not limited to patients on secondary prevention, actively managing treatment and optimal prescription with less treatment burden are also important for those on primary prevention.

Our study showed that LLT with concomitant medications for the treatment of depression/anxiety was associated with shorter persistence, whereas patients with medications for hypertension or gout were associated with better adherence and longer persistence. The finding of depression increasing the risk of discontinuation was consistent with the previous meta-analysis study. ${ }^{14}$ Anxiety and depression are most commonly reported as obstacles hindering the adoption of a healthier lifestyle as a result of emotional issues. ${ }^{29}$ A previous study suggested that patients with a healthy lifestyle are more adherent because they are more likely to seek for healthier behaviors, such as refilling prescriptions and visiting their physicians routinely. ${ }^{30}$

A previous survey in Japan revealed that patients on hypertensive medicine had a higher awareness of the necessity of taking medication appropriately as compared with those on antidyslipidemic medicine $(85.5 \%$ vs. $37.2 \%$, $\mathrm{P}<0.001) .{ }^{24}$ In clinical practice in Japan, routine and frequent measurement of blood pressure at home is highly recommended by the treatment guidelines, ${ }^{31}$ which may enhance disease awareness in patients being treated for hypertension. The finding of this study showing better adherence and persistence in patients with elevated blood cholesterol levels on antihypertensive medications may have resulted from the enhanced awareness of drug-taking behavior. At present, limited studies have revealed a direct association between adherence and LLT and gout therapy. Our results demonstrated that patients on LLT with concomitant medications for gout were more likely to be adherent to medications, which could result from increased awareness of treatment importance because of the severe gout pain experienced by patients.

Although a high number of concomitant pills was reported to be associated with poor adherence in previous studies, that factor was excluded from the current model by the stepwise selection procedure. Multiple reasons could explain this. First, the increasing number of concomitant pills highly reflects the number of comorbidities, which may lead to improved health literacy and awareness of diseases by individual patients. Second, $66 \%$ of our study patients were concomitantly using antihypertensive medication, which was previously reported as being associated with better adherence. Also, our additional analysis showed that pill number was associated with age (Supplementary Figure 2), where older age patients (i.e., 55-74 years old) were found to have better adherence.

Long-term persistence and adherence to LLT are essential for maximizing the clinical effectiveness for patients with dyslipidemia. Our findings found potential predictors resulting in poor adherence and persistence with statinezetimibe LLT. However, the follow-up period in our study was only 1 year and given that dyslipidemia is a chronic disease that requires life-time control, it is important to actively control dyslipidemia for decades for the prevention of CVD events. In particular, continuing medication management is crucial for those patients with relatively poorer adherence. For individuals requiring multiple LLTs with poor adherence and persistence, active interventions may improve adherence; for example, (1) long-term patient education program aimed at improving awareness of the negative effects of dyslipidemia and the significance of treatment; (2) reminder systems for medication adherence; (3) choosing a simple treatment regimen to reducing the patient's pill burden (e.g., medications with fixed-dose combination and/or less frequent dosing); and (4) explanation given to patients with a higher risk of poor adherence.

\section{Study Strengths and Limitations}

There are a number of strengths to our study. We used an administrative pharmacy claims database that collect dispensing records electronically and systematically, which could minimize patient recall bias compared with other data collection methods such as face-to-face interview or questionnaire. Unlike other hospital-based claims databases mostly from acute care hospitals in Japan, the database we used in our study covers out-of-hospital pharmacies and many prescription records, which may provide a comprehensive picture of drug utilization patterns, especially for non-acute conditions such as dyslipidemia.

Several limitations in our study should be considered when interpreting the study results. First, the actual intake of medication is neither trackable nor recorded in the database; thus, we cannot know if a patient takes the dispensed medications. Second, as there was a lack of diagnostic history and medical procedure records, dispensed medications were used as a proxy for estimating certain therapeutic area/comorbidities of patients, which can result in some misclassification bias. Third, because of the nature of database, we could not capture dispensing records taking place in uncontracted pharmacies.

\section{Conclusions}

To the best of our knowledge, this is the first study to focus on adherence of statin-ezetimibe combinational LLT in a real-world setting. Patients characterized as younger or older ( $<45,45-54, \geq 75$ years old) and on primary prevention were associated with poor adherence. Patients characterized as older ( $\geq 75$ years) and on primary prevention were associated with shorter persistence. Concomitant use of medications for hypertension and gout was associated 
with better adherence and persistence, and concomitant use of medications for depression/anxiety was associated with shorter persistence. Active interventions are required for patients with poor adherence and persistence.

\section{Acknowledgments}

The authors thank William Y.H. Kuan and other staff of IQVIA Solutions Japan K.K. for their substantial contributions to statistical analysis, and writing and editorial assistance, provided under contract with MSD K.K., Tokyo, Japan. This study was funded by MSD K.K., Tokyo, Japan. The sponsor was involved in the study design, data analysis, interpretation of the data, writing of the manuscript, and decision to submit the manuscript for publication.

\section{Disclosure Statement}

T.U. and S.T. own stock/stock options of Merck \& Co., Inc., Kenilworth, NJ, USA. A.H. and Y.P. are currently employed by AstraZeneca K.K. All other authors declare no conflicts of interest.

\section{References}

1. Japan Atherosclerosis Society. Guidelines for prevention of atherosclerotic cardiovascular disease: 2017 version. Tokyo: JAS, 2017.

2. Catapano AL, Graham I, De Backer G, Wiklund O, Chapman MJ, Drexel H, et al. 2016 ESC/EAS guidelines for the management of dyslipidaemias. Eur Heart J 2016; 37: 2999-3058.

3. Stone NJ, Robinson JG, Lichtenstein AH, Bairey Merz CN, Blum CB, Eckel RH, et al. 2013 ACC/AHA guideline on the treatment of blood cholesterol to reduce atherosclerotic cardiovascular risk in adults: A report of the American College of Cardiology/American Heart Association Task Force on Practice Guidelines. J Am Coll Cardiol 2014; 63: 2889-2934.

4. Mihaylova B, Emberson J, Blackwell L, Keech A, Simes J, Barnes EH, et al. The effects of lowering LDL cholesterol with statin therapy in people at low risk of vascular disease: Metaanalysis of individual data from 27 randomised trials. Lancet 2012; 380: $581-590$.

5. Baigent C, Blackwell L, Emberson J, Holland LE, Reith C, Bhala $\mathrm{N}$, et al. Efficacy and safety of more intensive lowering of LDL cholesterol: A meta-analysis of data from 170,000 participants in 26 randomised trials. Lancet 2010; 376: 1670-1681.

6. Caspard H, Chan AK, Walker AM. Compliance with a statin treatment in a usual-care setting: Retrospective database analysis over 3 years after treatment initiation in health maintenance organization enrollees with dyslipidemia. Clin Ther 2005; 27: $1639-1646$

7. Benner JS, Tierce JC, Ballantyne CM, Prasad C, Bullano MF, Willey VJ, et al. Follow-up lipid tests and physician visits are associated with improved adherence to statin therapy. Pharmacoeconomics 2004; 22(Suppl 3): 13-23.

8. Donnelly LA, Doney AS, Morris AD, Palmer CN, Donnan PT Long-term adherence to statin treatment in diabetes. Diabet Med 2008; 25: 850-855.

9. Benner JS, Glynn RJ, Mogun H, Neumann PJ, Weinstein MC, Avorn J. Long-term persistence in use of statin therapy in elderly patients. JAMA 2002; 288: 455-461.

10. Chowdhury R, Khan H, Heydon E, Shroufi A, Fahimi S, Moore $\mathrm{C}$, et al. Adherence to cardiovascular therapy: A meta-analysis of prevalence and clinical consequences. Eur Heart J 2013; 34: $2940-2948$

11. World Health Organization. Adherence to long-term therapies: Evidence for action. http://apps.who.int/iris/bitstream/handle/ 10665/42682/9241545992.pdf;jsessionid=1D314DB5F92665BFA 30324FE75A74DF7?sequence $=1$ (accessed April 19, 2018).

12. Haynes RB, McKibbon KA, Kanani R. Systematic review of randomised trials of interventions to assist patients to follow prescriptions for medications. Lancet 1996; 348: 383-386.

13. Tuppin P, Neumann A, Danchin N, de Peretti C, Weill A, Ricordeau $\mathrm{P}$, et al. Evidence-based pharmacotherapy after myocardial infarction in France: Adherence-associated factors and relationship with 30-month mortality and rehospitalization. Arch Cardiovasc Dis 2010; 103: 363-375.
14. Ofori-Asenso R, Jakhu A, Curtis AJ, Zomer E, Gambhir M, Jaana Korhonen M, et al. A Systematic review and meta-analysis of the factors associated with nonadherence and discontinuation of statins among people aged $\geq 65$ years. J Gerontol A Biol Sci Med Sci 2018; 73: 798-805.

15. Degli Esposti L, Saragoni S, Batacchi P, Benemei S, Geppetti P, Sturani A, et al. Adherence to statin treatment and health outcomes in an Italian cohort of newly treated patients: Results from an administrative database analysis. Clin Ther 2012; 34: 190-199.

16. Chodick G, Shalev V, Gerber Y, Heymann AD, Silber H, Simah $\mathrm{V}$, et al. Long-term persistence with statin treatment in a not-forprofit health maintenance organization: A population-based retrospective cohort study in Israel. Clin Ther 2008; 30: $2167-$ 2179.

17. Mann DM, Woodward M, Muntner P, Falzon L, Kronish I Predictors of nonadherence to statins: A systematic review and meta-analysis. Ann Pharmacother 2010; 44: 1410-1421.

18. Ministry of Health Labour and Welfare. Heisei 26 nen kanzya tyousa no gaikyou [translated title in English: Summary of patient survey in 2014 ]. The Ministry, 2014. http://www.mhlw. go.jp/toukei/saikin/hw/kanja/14/index.html (accessed April 4, 2018).

19. Raebel MA, Schmittdiel J, Karter AJ, Konieczny JL, Steiner JF. Standardizing terminology and definitions of medication adherence and persistence in research employing electronic databases. Med Care 2013; 51: S11-S21.

20. Pednekar P, Malmenas MM, Ágh T, Bennett B, Peterson A. Measuring multiple medication adherence: Which measure when? Value Outcomes Spotlight 2017; 3: 17-20.

21. Nau DP. Proportion of days covered (PDC) as a preferred method of measuring medication adherence. Pharmacy Quality Alliance.

22. Vinogradova Y, Coupland C, Brindle P, Hippisley-Cox J. Discontinuation and restarting in patients on statin treatment: Prospective open cohort study using a primary care database. BMJ 2016; 353: $\mathrm{i} 3305$.

23. Hippisley-Cox J, Coupland C. Unintended effects of statins in men and women in England and Wales: Population based cohort study using the QResearch database. BMJ 2010; 340: c2197.

24. Katada J, Hirai M, Takahashi S. Adherence and persistence of a single pill amlodipine/atorvastatin vs. two pill regimen based on Japanese health insurance claims. Prog Med 2012; 32: 1327-1335 (in Japanese).

25. Grant RW, O'Leary KM, Weilburg JB, Singer DE, Meigs JB Impact of concurrent medication use on statin adherence and refill persistence. Arch Intern Med 2004; 164: 2343-2348.

26. Chee YJ, Chan HH, Tan NC. Understanding patients' perspective of statin therapy: Can we design a better approach to the management of dyslipidaemia?: A literature review. Singapore Med J 2014; 55: 416-421.

27. Guglielmi V, Bellia A, Pecchioli S, Della-Morte D, Parretti D, Cricelli I, et al. Effectiveness of adherence to lipid lowering therapy on LDL-cholesterol in patients with very high cardiovascular risk: A real-world evidence study in primary care. Atherosclerosis 2017; 263: $36-41$

28. Cannon CP, Blazing MA, Giugliano RP, McCagg A, White JA, Theroux P, et al. Ezetimibe added to statin therapy after acute coronary syndromes. N Engl J Med 2015; 372: 2387-2397.

29. Khatib R, Schwalm JD, Yusuf S, Haynes RB, McKee M, Khan $\mathrm{M}$, et al. Patient and healthcare provider barriers to hypertension awareness, treatment and follow up: A systematic review and meta-analysis of qualitative and quantitative studies. PLoS One 2014; 9: e84238

30. Brookhart MA, Patrick AR, Dormuth C, Avorn J, Shrank W, Cadarette SM, et al. Adherence to lipid-lowering therapy and the use of preventive health services: An investigation of the healthy user effect. Am J Epidemiol 2007; 166: 348-354.

31. Japanese Society of Hypertension. Guidelines for the management of hypertension in 2014. Japanese Society of Hypertension, 2014 (in Japanese).

\section{Supplementary Files}

Please find supplementary file(s);

http://dx.doi.org/10.1253/circj.CJ-18-1344 